\title{
Reisemediziner sind überall dabei - auch im Weltraum
}
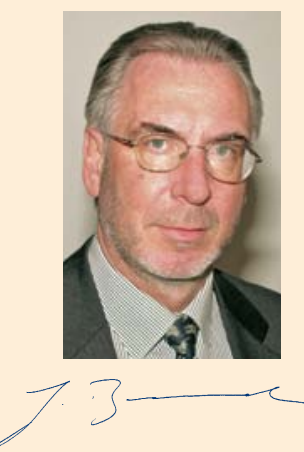

Liebe Kolleginnen, liebe Kollegen,

es hat sich nun so ergeben, dass ich als Tropenmediziner das Editorial schreibe für ein neues Heft der FTR, in dem es unter anderem um die Weltraummedizin geht.

Reisemedizin ist bekanntermaßen ein Querschnittsfach. Reisemediziner definieren gerne alles, was mit Mobilität von Menschen zu tun hat, als Reisemedizin (es gab einmal sehr schöne Kongresse „Medizin und Mobilität“). Und wenn Menschen ins Weltall reisen, gehört die Weltraummedizin auch dazu. Allerdings: Weltraummedizin beschäftigt sich in erster Linie mit den Problemen bei Langzeitaufenthalten im All. Das ist sicherlich sinnvoll, weil wir aus meiner Sicht die bemannte Raumfahrt benötigen. Zum einen ist es eine Kulturleistung, unsere Umgebung wissenschaftlich zu erforschen. Zum anderen geht es um die notwendige Erweiterung menschlicher Lebensräume außerhalb der Erde. Zitat NASA-Administrator Michael Griffin in der Washington Post am 25. September 2005: „If we humans want to survive for hundreds of thousands or millions of years, we must ultimately populate other planets. We may have people living on the moons of Jupiter and other planets. We may have people making habitats on asteroids. We've got places that humans will go, not in our lifetime, but they will go there.“ Da müssen wir Reisemediziner natürlich dabei sein!!!

Astronauten arbeiten ja in einer ziemlich lebensfeindlichen Umgebung, aber vielleicht kann man doch für die „normale“ Medizin etwas von der Weltraummedizin lernen. Manche Anpassungen des Körpers unter Schwerelosigkeit ähneln Symptomen im Alter. Abnahme der Knochendichte und Muskelschwund in der Schwerelosigkeit sind ähnlich wie bei längerer Bettlägerigkeit. Je länger ein Weltraumaufenthalt dauert, desto größer wird die psychische Belastung und desto eher zeigen sich Auswirkungen auf das vegetative Nervensystem. Und aus infektiologischer Sicht sind sicherlich die Veränderungen des Immunsystems interessant, zum Beispiel die durch Stress induzierte Veränderung der T-Zell-Subpopulationen (siehe FTR 2011; 18: 118-122: Funktion des Immunsystems in Schwerelosigkeit - Vom Astronauten für die Erde lernen). Und aus infektiologischer Sicht muss man sich auch Gedanken machen über den interplanetaren Transport von Mikroorganismen (das würde ich jetzt aber nicht mehr unter Reisemedizin subsumieren...). Also: Kurze touristische Ausflüge ins All erscheinen - jetzt einmal aus tropenmedizinischer Sicht - Geldverschwendung zu sein und damit fragwürdig, aber medizinische Untersuchungen an Astronauten sind wichtig. Und insofern ist der Artikel in diesem FTR-Heft - glaube ich - für alle interessant.

Andere Themen des Heftes sind Sportklettern und die Lepra. Zur Lepra möchte ich hier nur einen Satz anfügen: Man sollte nicht glauben, dass die Lepra ausgerottet ist. Wir sehen immer wieder importierte Fälle, insbesondere bei Migranten, zum Teil mit schweren Verläufen und Leprareaktionen.

Ich glaube also, dieses Heft enthält wieder hochinteressante Artikel, welche die ganze Bandbreite unserer Fachgebiete abdecken - so wie es in der FTR sein soll. Ich wünsche Ihnen viel Vergnügen bei der Lektüre.

Mit besten Grüßen

Ihr Prof. Dr. Gerd Burchard, Hamburg 